\title{
Baseline evaluation of infection prevention and control (IPC) in the context of Ebola virus disease (EVD) in nine healthcare facilities in the city of Conakry, Guinea
}

\author{
A Diallo $^{1 *}$, M Diallo $^{1}$, Y Hyjazi ${ }^{1}, \mathrm{R}$ Waxman ${ }^{2}$, T Pleah $^{2}$ \\ From 3rd International Conference on Prevention and Infection Control (ICPIC 2015) \\ Geneva, Switzerland. 16-19 June 2015
}

\section{Introduction}

As a means to contribute to the response to the Ebola virus disease (EVD) epidemic and support health system strengthening, Jhpiego, with funding from USAID, committed to strengthening health service capacity in IPC.

\section{Objectives}

Determine IPC performance level in selected health facilities.

Identify gaps in IPC performance.

\section{Methods}

The assessment was conducted in December 2014 and January 2015 in three teaching hospitals, comprising 66 services, and six communal medical centers (CMC). The main assessment tool was based on SBM-R performance standards for IPC. Data collection consisted of observation, interview and document review by a team of two evaluators per facility, for 2-3 days per facility.

\section{Results}

In the three national teaching hospitals, among 66 services, one service achieved $75 \%$ of performance standards, and eight achieved none. 33 of the services achieved below $30 \%$ of standards, while 17 scored between 31 and $49 \%$ and seven were performing at $50-74 \%$ of standards. In the $6 \mathrm{CMC}$, four were performing between 16 and $20 \%$ of standards, and two were performing at $35 \%$ and $37 \%$ respectively. Key reasons for poor performance included: 1) Insufficient knowledge in IPC, 2) lack of IPC materials and equipment, 2) non-observance of IPC procedures and norms, 3) poor management of IPC activities.

\section{Conclusion}

Key interventions included training of healthcare workers, provision of an initial stock of IPC materials, onsite follow-up. These actions will contribute to the improvement of healthcare services, and help to restore community confidence in the public health services.

\section{Disclosure of interest}

None declared.

\section{Authors' details}

'Jhpiego, Conakry, Guinea. ${ }^{2} J$ hpiego, Baltimore, MD, USA.

Published: 16 June 2015

doi:10.1186/2047-2994-4-S1-010

Cite this article as: Diallo et al:: Baseline evaluation of infection prevention and control (IPC) in the context of Ebola virus disease (EVD) in nine healthcare facilities in the city of Conakry, Guinea. Antimicrobial Resistance and Infection Control 2015 4(Suppl 1):010.

1Jhpiego, Conakry, Guinea

Full list of author information is available at the end of the article 